\title{
Intervención logopédica en un caso con discapacidad intelectual en la adolescencia tardía
}

\author{
Ismael García Pallas \\ Universidade da Coruña
}

\begin{abstract}
Resumen
La literatura científica ha puesto de manifiesto que las habilidades pragmáticas son una de las dimensiones del lenguaje que se encuentran afectadas en las personas con discapacidad intelectual. El objetivo principal del presente trabajo fue llevar a cabo una intervención centrada en las habilidades pragmáticas y en la autonomía de una adolescente tardía (17 años) con un trastorno del desarrollo intelectual. El trabajo se desarrolló a lo largo de 3 fases: una evaluación previa, la fase de intervención y, finalmente, una valoración de los resultados obtenidos tras la intervención. Los resultados muestran una mejora en las habilidades pragmáticas relacionadas con las habilidades conversacionales, así como un incremento de vocabulario. Por otra parte, también se observaron mejoras en los distintos objetivos marcados con el fin de mejorar su autonomía.

Palabras clave: discapacidad intelectual, habilidades pragmáticas, estimulación lenguaje oral.
\end{abstract}

\section{Introducción}

La discapacidad intelectual (trastorno del desarrollo intelectual) se define actualmente como un trastorno que empieza durante el período de desarrollo y que incluye limitaciones del funcionamiento intelectual y del comportamiento adaptativo en los dominios conceptual, social y práctico (APA, 2014).

Presenta una prevalencia global en la población general de aproximadamente el $1 \%$, modificándose las tasas según la edad. En cuanto a la discapacidad intelectual grave las cifras se sitúan en seis de cada mil personas. Respecto al género, los varones presentan mayor tendencia que las mujeres de ser diagnosticados de formas de discapacidad intelectual leve (1,6: 1). En los casos de discapacidad intelectual grave la probabilidad se reduce (1,2: 1). Aunque estos datos son de estudios recientes, es importante señalar que existen variaciones dependiendo de las distintas publicaciones (APA, 2014). Lo cierto es que esta problemática ha estado presente a lo largo de toda la historia de la humanidad. Las dudas y polémicas surgen a la hora de implantar un término que defina dicho trastorno. A lo largo del tiempo, se han utilizado infinidad de términos para definirlo. De esta forma, antes del siglo XIX, el trastorno del desarrollo intelectual no se diferenciaba de otras problemáticas, siendo considerado como una variante de la demencia. En 1818, es cuando se distingue por primera vez entre "idiota” y "demencia” gracias a Esquirol, quien durante el siglo XIX define el retraso mental como un déficit mental constatable de origen orgánico (Sola Martínez, 2005). A partir del siglo XIX ya se comienzan a realizar diferencias entre la demencia y el resto de patologías. En el siglo XX, la Asociación Americana sobre personas con Retraso Mental (AAMR) (anteriormente Asociación Americana de Deficiencia Mental -AAMD-) abrió un camino hacia una definición más unificada, aunque revisándola y modificándola en varias ocasiones a lo largo del tiempo hasta llegar a la actualidad.

Respecto a la etiología, habitualmente se habla de causas orgánicas y genéticas o ambientales. Respecto a las primeras, se suelen mencionar cinco principales (Artigas-Pallarés, 2006): factores que actúan antes de la concepción, factores prenatales, perinatales, postnatales y causa desconocida.

Actualmente existe una clasificación respecto a la severidad del trastorno, pudiendo ser leve (personas con CI entre 50-55 y aproximadamente 70); moderado (CI entre 35-40 y 50-55); grave (CI entre 20-25 y 35-40) o profundo (CI inferior a 20-25) (APA, 2014; Artigas-Pallarés, 2006).

Por otro lado, cabe destacar que la intervención en estas personas persigue el objetivo de capacitar y preparar a los sujetos para la vida diaria en la medida de lo posible. Para ello, se intentarán trabajar todas aquellas áreas deficitarias priorizando objetivos en caso de no poder alcanzar los valores esperados en cada una de ellas. A continuación, se describen algunas de las posibles áreas afectadas y que se consideran de intervención prioritaria: área perceptivo-motriz, educación sexual, desarrollo cognitivo, adquisición de conceptos matemáticos, educación de conductas sociales/ prácticas y del lenguaje.

El objetivo principal del presente trabajo fue comprobar la efectividad de una intervención logopédica centrada en las habilidades pragmáticas, en concreto, las relacionadas con interacción, interacción social y habilidades conversacionales en una adolescente con discapacidad intelectual. Con la intervención se pretendía, además, incrementar el vocabulario y se planificaron actividades dirigidas a mejorar la autonomía personal y fortalecer el trabajo realizado en la Asociación a la que acudía la adolescente. A continuación se describe de manera precisa el desarrollo del trabajo y los resultados obtenidos tras la intervención.

\section{Método}

\section{Participantes}

En este estudio participó una joven de 17 años de edad (de aquí en adelante R.) con un trastorno del desarrollo intelectual que acudía a un instituto público situado en la 
localidad de Laxe, perteneciente a la provincia de la Coruña. Vive con sus dos padres, cuya madre presenta una posible discapacidad intelectual y el padre viaja continuamente debido a su empleo como marinero. También es oportuno señalar que a escasos metros vive su abuela, con quién R. pasa las tardes viendo la tele, por lo que se trata de un contexto poco estimulante.

En la actualidad cursa $2^{\circ}$ de Educación Secundaria Obligatoria, y tiene una adaptación curricular cuyo nivel es del primer ciclo de primaria en lengua gallega y castellana, matemáticas, ciencias naturales y sociales, inglés, educación física, tecnología y música. Además, dos días a la semana, acudía a la asociación Íntegro en Cabana de Bergantiños. Dicha entidad, busca la independencia y autonomía personal de los jóvenes que allí acuden. Para ello, realizan diferentes actividades como excursiones, manualidades, cultivan un pequeño huerto, jornadas de belleza y cuidado personal, deportes adaptados etc.

El centro de logopedia que realiza la intervención con R. en el área lingüística desplazándose al ayuntamiento de Laxe, asume como prioritarios dichos objetivos. Por ello, colabora con la asociación en la consecución de los mismos, además de centrar la intervención logopédica en el área pragmática. R. acudía dos días a la semana a los servicios de logopedia.

Los datos que facilitó el colegio acerca de las dificultades que presentaba la joven indicaban la existencia de un notable retraso del desarrollo intelectual, del cual no se dispone de informe para conocer su categoría, aunque está confirmada la concesión de un certificado de minusvalía psíquica.

En cuanto a las limitaciones que refieren, se apreciaban dificultades en las interacciones de carácter social y conversacional, problemas de memoria, atención y dificultades académicas muy notables respecto a los jóvenes de su edad.

\section{Instrumentos}

La evaluación se llevó a cabo a lo largo de dos fases; la primera correspondió con la evaluación previa a la intervención y, la segunda, con la evaluación posterior a la intervención.

En la fase de evaluación previa a la intervención, se utilizaron tres instrumentos. El Test Breve de Inteligencia de Kaufman (K-BIT) (Kaufman, 1997), Boehm Test de Conceptos Básicos (Boehm, 2000) y el Cuestionario de Registro de la Pragmática, incluido en el material de Evaluación de la Comunicación y el Lenguaje (ECOL) (Alemán, Ardanaz, Echeverría, Poyo y Yoldi, 2006). Luego, se realizó una evaluación informal mediante la construcción en cartulina de un supermercado.

\section{Procedimiento}

El presente trabajo se llevó a cabo a lo largo de dos meses. La fase de evaluación previa a la intervención, se desarrolló a lo largo de tres sesiones de treinta minutos cada una, en un aula habilitada para los servicios logopédicos en el interior del ayuntamiento de Laxe. Dicha aula reunía las condiciones idóneas respecto a la iluminación, acústica y temperatura. Además, contaba con el mobiliario necesario para desarrollar la evaluación sin ningún tipo de problema.

Antes de iniciar las sesiones de evaluación y con el objetivo de garantizar un buen clima y desarrollar un vínculo de confianza con la joven, se llevó a cabo una breve presentación y se realizaron algunas sesiones lúdicas con la ayuda de su logopeda habitual para facilitar su integración a la nueva situación. Una vez realizadas dichas sesiones, se comenzó con la aplicación del Boehm Test de Conceptos Básicos (Boehm, 2000) durante una sesión de treinta minutos. La joven mostró una actitud colaboradora en todo momento, queriéndose anticipar incluso antes de terminar de dar las órdenes.

En la segunda sesión de evaluación, se aplicó el Test Breve de Inteligencia de Kaufman (K-BIT) (Kaufman, 1997). En cuanto a la actitud de la adolescente, es importante destacar que seguía siendo magnífica, aunque mostraba cansancio según se iba avanzando en los ítems. Finalmente, en la tercera sesión se utilizó el material preparado para comprobar las capacidades matemáticas en la suma y en el manejo de las monedas. Debido a la dificultad que le suponía la utilización de las monedas, se utilizaron únicamente monedas de un euro en un primer momento, luego dos euros $\mathrm{y}$, para terminar, ambas monedas.

En función de los resultados obtenidos tras la fase de evaluación, se diseñó un plan de intervención con dos objetivos principales: mejorar las habilidades pragmáticas, en concreto, interacción, interacción social y habilidades conversacionales; e incrementar el vocabulario. Además, otros objetivos que se trabajaron para reforzar el trabajo realizado por la asociación Íntegro con el fin de potenciar su autonomía.

Las actividades dirigidas a potenciar su autonomía se centraron principalmente en el manejo de los euros, el empleo de la calculadora para tal fin y el manejo del teléfono. Se seleccionaron los anteriores objetivos específicos con el fin de facilitar la vida diaria de la joven y de sus familiares, ya que frecuentemente la situación familiar exigía dichas tareas.

Respecto a las habilidades conversacionales, se establecieron como objetivos específicos mejorar el contacto ocular y la utilización de saludos y formas de cortesía en los momentos adecuados.

En cuanto a las actividades diseñadas para incrementar el vocabulario, se centraron principalmente, en las nociones espaciales, ya que era el ámbito más dañado a nivel semántico. Aun así, para ejercitar dicho objetivo, se utilizó gran cantidad de vocabulario organizado por campos semánticos con la finalidad de ayudar a la joven a recordar y aprender conceptos nuevos en caso de desconocimiento de los mismos.

En la tabla 1 se recogen los objetivos generales y específicos propuestos para la intervención.

La planificación de la intervención se organizó teniendo en cuenta el horario que estaba establecido con anterioridad por su logopeda habitual. Se realizaban dos sesiones semanales de treinta minutos. Es importante recordar que la adolescente asistía también a la asociación Î́ntegro y al instituto, por lo que más sesiones o de mayor duración podían ocasionarle un cansancio excesivo. 
Tabla 1.

Objetivos de la intervención

\section{Objetivo general}

Potenciar la autonomía

$$
\begin{aligned}
& \text { Objetivo específico } \\
& \text { Manejo de los euros } \\
& \text { - } \quad \text { Diferenciar monedas } \\
& \text { de } 1 \text { y } 2 € \\
& \text { - } \quad \text { Empleo de monedas } \\
& \text { de } 1 € \\
& \text { - } \quad \text { Empleo de monedas } \\
& \text { de } 2 € \\
& \text { Empleo de ambas } \\
& \text { monedas }
\end{aligned}
$$

Empleo de la calculadora

- Encender

- Apagar

- Seleccionar números

- Sumar

Empleo del teléfono

- Descolgar

- Marcar

- Hilo conversacional

Potenciar habilidades conversacionales

Empleo de saludos y formas de cortesía

- Saludo

- Despedida

- Situaciones espontáneas

- Contacto ocular

Incremento vocabulario

Nociones

espaciales/temporales

- Campos semánticos

Durante las ocho sesiones, se trabajaron todos los objetivos marcados en el plan de intervención. Se comenzaba por las actividades relacionadas con el uso de las monedas por dos motivos principales. Por una parte, manipular y observar las monedas producía una gran motivación en la adolescente. Por otra parte, se intentaba aprovechar que al principio de la sesión su nivel de cansancio era menor. Según pasaban los minutos, se notaba mayor agotamiento e inatención, por lo que era preferible cambiar de actividad y dejar para el final actividades más relajadas: empleo del teléfono, nombrar objetos, distribuirlos por la clase, etc.

Siempre se trabajaban varios objetivos durante cada sesión con el fin de mantener la motivación y atención. También es importante señalar que en muchas ocasiones el uso de saludos y formas de cortesía así como el contacto ocular no se trabajaban de forma independiente, sino que se intentó trabajar durante la realización de otros objetivos tales como ser dependienta de un supermercado, el empleo del teléfono o la propia situación de llegada y despedida de la joven cuando llegaba al aula. Por otra parte, también se añadió el objetivo de usar la calculadora (paralelamente al uso de las monedas) con el fin de facilitarle la suma $y$, de este modo, servirle en su vida cotidiana para realizar pequeños recados en un súper cercano a su casa. Es importante señalar que si no emplea la calculadora necesita un gran periodo de tiempo para sumar y, a veces, comete errores.

En cuanto a la dificultad de las actividades, se intentó progresar en dificultad según iban pasando las sesiones. En caso de que alguna actividad se le complicase más de lo previsto, en la sesión posterior se planteaba algún ejercicio igual o similar con un fin más sencillo. Respecto al estilo de actividades, siempre se tuvieron en cuenta los gustos de la adolescente. En su vida diaria solía acompañar a sus padres a comprar, mostrando una especial ilusión cada vez que se trabajaba en el aula con actividades similares (ejercer de dependienta, de clienta...). De esta forma, las actividades se planteaban intentando buscar la máxima motivación y atención.

En cuanto al tiempo empleado, siempre se realizaban todas las actividades propuestas en cada una de las sesiones, ya que intercalar actividades resultaba realmente interesante para disminuir la fatiga y aburrimiento.

En todas las sesiones llevadas a cabo, la actitud y disposición de la niña a la hora de hacer las tareas fue inmejorable. Aunque tenía sus preferencias, siempre participaba en todas las actividades propuestas muy animada. Cada vez que realizaba una tarea, se le reforzaba verbalmente valorando su esfuerzo más allá de que lo realizara de manera exitosa o no. Habitualmente, respondía con una sonrisa y manifestaba que no estaba cansada. En caso de cometer errores, se le indicaba cuáles eran los mismos y se procuraba ponerle ejemplos para facilitar su comprensión.

\section{Resultados}

A continuación se exponen los resultados obtenidos en cada una de las pruebas empleadas tanto en la fase previa a la intervención como una vez finalizada la misma.

En primer lugar señalar que los datos obtenidos con el test Breve de Inteligencia de Kaufman (K-BIT) (Kaufman, 1997), confirmaron que R. presenta un trastorno del desarrollo intelectual. Concretamente la joven alcanza un CI de 46, por lo que, atendiendo a los criterios del DSM-5 (APA, 2014), los datos apuntan a la presencia de un trastorno del desarrollo intelectual moderado. Respecto a los resultados de las subpruebas, R. alcanza un CI de 45 en vocabulario y 65 en matrices. De este modo refleja un retraso moderado respecto al vocabulario y un retraso leve en cuanto a las matrices.

En segundo lugar se describen los resultados obtenidos con el Boehm Test de Conceptos Básicos (Boehm, 2000), comparando el número de aciertos obtenidos en ambas fases de evaluación. En la fase de evaluación previa a la intervención se obtuvieron 19 aciertos en el cuadernillo A. En el cuadernillo B los resultados alcanzan los 9 aciertos. Analizando la fase de evaluación posterior a la intervención, los resultados obtenidos son de 20 aciertos en el cuadernillo A y 16 en el cuadernillo 
B. Como resultados finales, de los 50 ítems mostrados R. alcanzó con éxito 28 en la evaluación previa y 36 en la postevaluación. Además, señalar que en la evaluación previa a la intervención se detectaron errores mayoritariamente en las nociones espaciales y de cantidad, por lo que la intervención se enfocó en dichas nociones tal y como se aprecia en la figura 1. Aun así, se intentó trabajar las partes más afectadas sin descuidar el resto de la semántica.

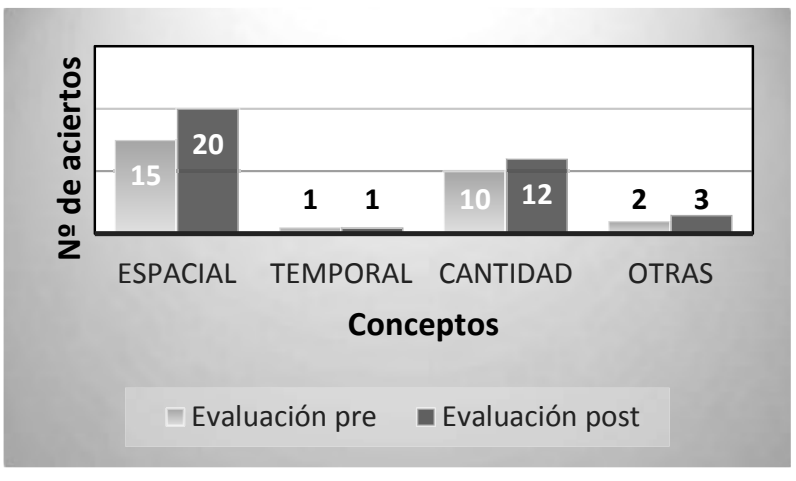

Figura 1. Número de aciertos en las categorías del Boehm Test en ambas fases de evaluación.

Como se puede comprobar en la figura 1, se produjo un incremento en el número de aciertos en todas las categorías una vez realizada la intervención, excepto en la de nociones temporales en la que no se produjeron cambios. El incremento más significativo tuvo lugar en la categoría de conceptos espaciales, pasando de 15 aciertos en la fase de evaluación previa a la intervención a 20 una vez finalizada ésta. En la evaluación previa R. consiguió una puntuación centil de 15, la cual corresponde al desempeño de niños/as de cuatro años en el primer trimestre. Respecto a la evaluación realizada tras la intervención R. alcanza una puntuación centil de 40, lo que refleja una clara mejoría respecto a la evaluación previa. No obstante, debe tenerse en cuenta que estos resultados se equiparan a los obtenidos por niños del segundo curso de enseñanza infantil (4 años) en el primer trimestre.

A continuación se comparan los resultados con el Cuestionario de Registro de la Pragmática, incluido en el material de Evaluación de la Comunicación y el Lenguaje (ECOL) (Alemán, et al., 2006), en ambas fases de evaluación. Es oportuno señalar que los resultados obtenidos con el cuestionario ECOL son de tipo cualitativo, pero en este caso se ha realizado una adaptación respecto a su corrección, con el fin de otorgar a estos resultados un valor cuantitativo, de manera que facilitase la descripción e interpretación de los resultados. De este modo, se concedió a cada ítem un valor numérico 1 o 0 , de manera que 1 reflejaba que $\mathrm{R}$. realizaba lo que el ítem indicaba, mientras que una puntuación de 0 se otorgaba a aquellos ítems en los que R. no realizaba lo indicado por el ítem o en los casos en los que reflejaba aspectos negativos de las funciones pragmáticas y habilidades conversacionales.
Una vez recogida toda la información de las subpruebas evaluadas con el ECOL, se realizó una comparación entre la puntuación total obtenida en las fases pre y post intervención. En función de los resultados obtenidos en la evaluación previa a la intervención, se observó con respecto a las funciones pragmáticas, que R. obtenía puntuaciones más bajas en las áreas de interacción, interacción social y metacognitiva. En el resto de subpruebas los resultados obtenidos en la evaluación previa a la intervención indicaban un buen nivel de R. con respecto a habilidades pragmáticas en las áreas de regulación, información, personal y diálogo, manteniéndose estos resultados tras la intervención. Por lo que se refiere a las habilidades conversacionales, en todas las subpruebas (organización formal de la conversación, mantenimiento del significado y adaptación a los interlocutores) R. obtiene puntuaciones bajas teniendo en cuenta su edad. Teniendo en cuenta estos resultados, y en función de los objetivos terapéuticos fijados se decidió que serían objeto de intervención, las áreas de interacción, interacción social y adaptación a los interlocutores. En este sentido, se observa una clara mejoría en la interacción (dar y recibir), en la interacción social (empleo de saludos y formas de cortesía) y en la adaptación a los interlocutores perteneciente a las habilidades conversacionales. Concretamente se pasa de 3, 2, 10 ítems correctos en la evaluación previa a 7, 9, 14 en la evaluación posterior respectivamente.

Finalmente, comentar los datos recogidos mediante la evaluación informal llevada a cabo con el supermercado diseñado para este fin. En la evaluación previa se detectaron una serie de problemas que se describen a continuación. En primer lugar, se apreciaban severas dificultades para realizar las sumas con los precios de los diferentes productos. Necesitaba una gran cantidad de tiempo para lograrlo y, a mayores, mostraba inseguridad.

Por otra parte, no manejaba adecuadamente la calculadora, necesitando ayuda en todo momento para sumar, encender y apagar. Una vez establecida la suma aparecían los problemas para distinguir las monedas y su valor.

Debido a las dificultades para sumar, se pensó que la utilización de la calculadora le ayudaría a ganar seguridad, no sufrir equivocaciones y disminuir el tiempo empleado. Por este motivo se trabajó su empleo durante las sesiones de intervención.

Tras la intervención R. no presentó dificultades para manejar la calculadora, aprendiendo a encender, apagar, borrar en caso de error, sumar y marcar los números pertinentes. En segundo lugar, también consiguió diferenciar las monedas de uno y dos euros, aunque persisten los problemas para entender su valor. Si se le presentan monedas del mismo valor es capaz a sumarlas y dar el valor correcto. Si se le presenta un puñado de monedas de 1 y 2 euros mezcladas, se manifiestan las dificultades para entender que su valor es distinto.

Otras de las actividades realizadas en ambas evaluaciones fueron la realización de llamadas ficticias. En la evaluación previa ya se observaba un buen manejo del teléfono, aunque las dificultades se evidenciaban en la forma de expresarse, saludos, despedidas y en el 
recuerdo de la información tratada. En la evaluación posterior a la intervención se logró realizar de manera exitosa todos estos aspectos a excepción de las dificultades para recordar toda la información proporcionada en cada llamada.

Finalmente mencionar que otra de las habilidades valoradas fue el contacto ocular por parte de R. en las interacciones comunicativas. En la evaluación previa se observó cómo R. rechazaba dicho contacto, huyendo con la mirada. Aunque se realizaron ejercicios específicos para tal fin, lo cierto es que la joven apenas mejoró en este objetivo. En la evaluación final R. tampoco era capaz de mirar a los ojos y, si se le indicaba que debía hacerlo, miraba y apartaba la mirada rápidamente.

\section{Discusión y conclusiones}

El objetivo principal del presente trabajo fue llevar a cabo una intervención logopédica con una adolescente que presentaba un trastorno del desarrollo intelectual para mejorar las habilidades pragmáticas, en concreto, interacción, interacción social y habilidades conversacionales; e incrementar el vocabulario. Además se pretendía mejorar su nivel de autonomía. El desarrollo de estas habilidades repercutiría de manera secundaria en la mejora de la calidad de vida de la paciente y su familia, favoreciendo la integración social, además de desarrollar habilidades útiles y prevenir el deterioro cognitivo.

Los resultados obtenidos concuerdan con los obtenidos en los trabajos en los que se ponía de manifiesto que existen varios aspectos del trastorno del desarrollo intelectual que pueden repercutir negativamente en el desarrollo de la pragmática y provocar dificultades en las situaciones de interacción (Monfort, Juárez Sánchez, \& Monfort Juárez, 2004). Por otra parte, este estudio también revela las posibles limitaciones en la capacidad de aprendizaje que se pueden hacer visibles en aspectos como el léxico, reduciendo la amplitud y variabilidad de recursos así como dificultades para realizar ciertas operaciones mentales como por ejemplo tener en cuenta la información de los demás, manejar varias informaciones en paralelo, etc.

A la hora de interpretar los resultados es necesario tener en cuenta que la joven presentaba un trastorno del desarrollo intelectual moderado, por lo que la capacidad de aprendizaje y las distintas capacidades cognitivas van a repercutir negativamente, tal y como demuestran los estudios anteriormente citados.

Otro de los aspectos de probable influencia en los resultados, es el entorno de la adolescente. Al finalizar las sesiones de intervención logopédica, R. pasaba el resto de la tarde en casa de su abuela mirando el televisor. Cabe recordar también que su madre sufría una posible discapacidad intelectual y que el padre era marinero, por lo que pasaba bastantes horas fuera de casa. En referencia a esto, existen estudios que manifiestan respecto a los factores ambientales una inhibición de muchas de las habilidades pragmáticas en las personas con trastorno del desarrollo intelectual impidiendo así la adquisición de éstas y su posterior uso (Hatton, 1998).

Entre las recomendaciones a seguir en el trabajo logopédico con R. señalar que la intervención debería mantenerse a largo plazo procurando trabajar todos los objetivos fijados en el plan de intervención y tratando de fortalecer los ya alcanzados.

Finalmente, se debería llevar a cabo un seguimiento más largo para comprobar la evolución de R., además de una evaluación más exhaustiva a nivel de capacidades cognitivas tales como memoria, atención y razonamiento para tratar de incorporarlas a la intervención en función de los resultados obtenidos.

\section{Referencias}

Alemán, N., Ardanaz, J., Echeverría, A., Poyo, D., y Yoldi, S. (2006). Evaluación de la Comunicación y del Lenguaje (ECOL). Navarra: Fondo de Publicaciones del Gobierno de Navarra.

Almenara Barrios, J., García González-Gordon, R., Novalbos Ruiz, J. P., Merello Martel, B., Abellan Hervás, M. J., \& García Ortega, C. (1999). Evaluación médica y psicosocial de una población adulta con discapacidad intelectual. Revista Española De Salud Pública, 73(3), 383-392.

American Psychiatric Association -APA-. (2014). Manual diagnóstico y estadístico de los trastornos mentales: DSM-5 ( $5^{\mathrm{a}}$ ed.). Buenos Aires: Panamericana..

Artigas-Pallarés, J. (2006). Tratamiento farmacológico del retraso mental. Revista de Neurología, 42 (Supl 1), S109-S115.

Boehm, A. E. (2000). Boehm test de conceptos básicos (10 a ed.). Madrid: TEA.

Hatton, C. (1998). Pragmatic language skills in people with intellectual disabilities: A review. Journal of Intellectual and Developmental Disability, 23(1), 79-100.

Kaufman, A. S. (1997). K-BIT: Test breve de inteligencia de kaufman. Madrid: TEA.

Monfort, M., Juárez Sánchez, A., \& Monfort Juárez, I. (2004). Niños con trastornos pragmáticos del lenguaje y de la comunicación ndescripción e intervención. Madrid: Entha. 56-76.

Sola Martínez, T. (2005). Análisis e intervención educativa en la deficiencia mental. Granada: Grupo Editoral Universitario. 